\title{
Discontinuity-capturing operators for elastodynamics
}

\author{
Gregory M. Hulbert \\ Department of Mechanical Engineering and Applied Mechanics, The University of Michigan, \\ Ann Arbor, MI 48109-2121, USA \\ Received 10 August 1990 \\ Revised 22 March 1991
}

\begin{abstract}
Finite element methods are presented which include discontinuity-capturing operators to accurately solve elastodynamics problems exhibiting sharp gradients in their solution, e.g., wave propagation. The formulation involves finite element discretization of the temporal domain as well as the usual finite element discretization of the spatial domain. Linear least-squares terms are included that enhance the stability of the space-time finite element method. Nonlinear discontinuity-capturing operators are presented that result in more accurate capturing of wave fronts in transient solutions while maintaining the high-order accuracy of the underlying linear algorithm in smooth regions. Stability of the discontinuity-capturing formulation is proved and a convergence proof is given for a particular choice of the discontinuity-capturing operator. Numerical results are presented that demonstrate the effectiveness of the proposed discontinuity-capturing operators for elastodynamics.
\end{abstract}

\section{Introduction}

The development of algorithms to solve problems that exhibit discontinuities (shocks) or sharp gradients in the solution principally has focused on problems in fluid mechanics. Numerous problems in solid mechanics also require accurate capturing of discontinuities, e.g., wave propagation. The classical approach towards capturing discontinuities is to introduce a shock viscosity in an algorithm. Since the fundamental work of Von Neumann and Richtmyer [1], numerous shock viscosities have been proposed within the finite difference community for computational fluid mechanics. While successful algorithms have been developed for onedimensional flows, extending these formulations to the multidimensional case has been a major challenge for the last 40 years. Christensen has recently developed an effective multidimensional viscosity called the flux-limited shock viscosity. Benson [2] has extended the flux-limited shock viscosity to solve solid mechanics problems using a multidimensional Lagrangian formulation on an arbitrary mesh. (A good overview of shock viscosities using the finite difference approach is given in [2].)

New finite element methods for computational fluid dynamics have been developed that also are capable of accurately capturing shocks. The work of Hughes et al. [3] and Hughes and Mallet [4] introduced effective shock-capturing operators within the context of finite element formulations for fluid mechanics. Johnson and Szepessy [5-8] and Galeão and Dutra do Carmo $[9,10]$ have further advanced the development of shock-capturing operators for first-order hyperbolic systems. A general framework for the design of discontinuity-capturing operators has been developed by Shakib [11] for the Navier-Stokes equations. One advantage of these finite element methods, compared to finite difference methods, is that from the outset they are formulated for multidimensional problems. Also, the finite element formulations are 
more amenable to mathematical analysis, e.g., Johnson and Szepessy [5-8] have proved convergence of their discontinuity-capturing formulations for Burger's equation. Their studies are interesting as they show that discontinuity-capturing operators are needed to prove convergence for problems exhibiting sharp gradients.

This paper presents a finite element formulation for solving elastodynamics problems; discontinuity-capturing operators are included to accurately resolve wave fronts. The underlying foundation of the proposed formulation is the space-time finite element method in which both the temporal and spatial domain are discretized using finite elements. Linear leastsquares terms are included that enhance the stability of the space-time finite element method. The space-time Galerkin/least-squares method for elastodynamics was first presented in [12]; stability and convergence of the formulation was proved for arbitrary polynomials in space and time. For wave propagation problems, nearly oscillation-free solutions are computed using the space-time Galerkin/least-squares method; a few oscillations occur near the wave front. The nonlinear discontinuity-capturing operators presented in this paper are designed to control these oscillations without degrading the accuracy of the underlying Galerkin/least-squares method in smooth regions of the solution.

In Section 2, a brief review of the equations of linear elastodynamics is given. 'Ihe space-time Galerkin/least-squares method is presented in Section 3. In Section 4, the design of discontinuity-capturing operators is discussed and two choices for these operators are given. A stability proof and derivation of error estimates for a particular choice of the discontinuitycapturing operator are presented in Section 5. Numerical results are given in Section 6 to demonstrate the effectiveness of the proposed discontinuity-capturing algorithms. Finally, conclusions are drawn in Section 7.

\section{Classical linear elastodynamics}

Consider an elastic body occupying an open, bounded region $\Omega \subset \mathbb{R}^{d}$, where $d$ is the number of space dimensions. The boundary of $\Omega$ is denoted by $\Gamma$. Let $\Gamma_{g}$ and $\Gamma_{h}$ denote non-overlapping subregions of $\Gamma$ such that

$$
\Gamma=\overline{\Gamma_{g} \cup \Gamma_{h}} .
$$

The displacement vector is denoted by $\boldsymbol{u}(\boldsymbol{x}, t)$, where $\boldsymbol{x} \in \bar{\Omega}$ and $t \in[0, T]$, the time interval of length $T>0$. The stress is determined by the generalized Hooke's law:

$$
\boldsymbol{\sigma}(\nabla \boldsymbol{u})=\boldsymbol{c} \cdot \nabla \boldsymbol{u}
$$

or, in components,

$$
\sigma_{i j}=c_{i j k l} u_{k, l}
$$

where $1 \leqslant i, j, k, l \leqslant d, u_{k, l}=\partial u_{k} / \partial x_{l}$; summation over repeated indices is implied. The elastic coefficients $c_{i j k l}=c_{i j k l}(\boldsymbol{x})$ are assumed to satisfy the following conditions:

$$
\begin{array}{ll}
c_{i j k l}=c_{j i k l}=c_{i j l k} & \text { (minor symmetries) } \\
c_{i j k l}=c_{k l i j} & \text { (major symmetry) }, \\
c_{i j k l} \psi_{i j} \psi_{k l}>0 \quad \forall \psi_{i j}=\psi_{j i} \neq 0 & \text { (positive definiteness) }
\end{array}
$$


The equations of the initial/boundary-value problem of elastodynamics are

$$
\begin{array}{ll}
\rho \ddot{u}=\nabla \cdot \sigma(\nabla u)+f & \text { on } Q \equiv \Omega \times] 0, T[, \\
\boldsymbol{u}=\boldsymbol{g} & \text { on } \left.Y_{g} \equiv \Gamma_{\boldsymbol{g}} \times\right] 0, T[, \\
\boldsymbol{n} \cdot \boldsymbol{\sigma}(\nabla u)=\boldsymbol{h} & \text { on } \left.Y_{\boldsymbol{h}} \equiv \Gamma_{h} \times\right] 0, T[, \\
\boldsymbol{u}(\boldsymbol{x}, 0)=\boldsymbol{u}_{0}(x) & \text { for } x \in \Omega, \\
\dot{u}(x, 0)=v_{0}(x) & \text { for } x \in \Omega,
\end{array}
$$

where $\rho=\rho(x)>0$ is the density, a superposed dot indicates partial differentiation with respect to $t, f$ is the body force, $g$ is the prescribed boundary displacement, $h$ is the prescribed boundary traction, $\boldsymbol{u}_{0}$ is the initial displacement, $\boldsymbol{v}_{0}$ is the initial velocity and $n$ is the unit outward normal to $\Gamma$. The objective is to find a $u$ that satisfies (7)-(11) for given $\rho, \boldsymbol{c}, \boldsymbol{f}, \boldsymbol{g}, \boldsymbol{h}$, $\boldsymbol{u}_{0}$ and $\boldsymbol{v}_{0}$.

REMARK. The form of the generalized Hooke's law, (2), was chosen as it enables a generalization of the proposed finite element formulation for nonlinear elastodynamics where the first Piola-Kirchhoff stress, $\boldsymbol{P}$, replaces $\boldsymbol{\sigma}$ (see [13]).

\section{A space-time Galerkin/least-squares finite element formulation}

\subsection{Preliminaries}

Consider a partition of the time domain, $I=] 0, T$, having the form: $0=t_{0}<t_{1}<\cdots<$ $t_{N}=T$. Let $\left.I_{n}=\right] t_{n-1}, t_{n}\left[\right.$ and $\Delta t_{n}=t_{n}-t_{n-1}$. Referring to Fig. 1 , the following notations are employed:

$$
\begin{aligned}
& Q_{n}=\Omega \times I_{n}, \\
& Y_{n}=\Gamma \times I_{n}, \\
& \left(Y_{g}\right)_{n}=\Gamma_{g} \times I_{n}, \\
& \left(Y_{h}\right)_{n}=\Gamma_{h} \times I_{n} .
\end{aligned}
$$

$Q_{n}$ is referred to as the $n$th space-time slab.

Let $\left(n_{\mathrm{el}}\right)_{n}$ denote the number of space-time elements in $Q_{n} ; Q_{n}^{e} \subset Q_{n}$ denotes the interior of the eth element; $Y_{n}^{e}$ denotes its boundary. Let

$$
\begin{aligned}
Q_{n}^{\Sigma} & =\bigcup_{e=1}^{\left(n_{\mathrm{el}}\right)_{n}} Q_{n}^{e} \quad \text { (element interiors), } \\
Y_{n}^{\Sigma} & =\bigcup_{e=1}^{\left(n_{\mathrm{el}}\right)_{n}} Y_{n}^{e}-Y_{n} \quad \text { (interior boundary). }
\end{aligned}
$$

Inner products over domain $D$ are denoted by $\left(\boldsymbol{w}^{h}, \boldsymbol{u}^{h}\right)_{D}$; the strain-energy inner product is denoted by $a(., .)_{\Omega}\left(a(., .)_{Q_{n}}\right.$ is the strain-energy inner product over the $n$th space-time slab). 

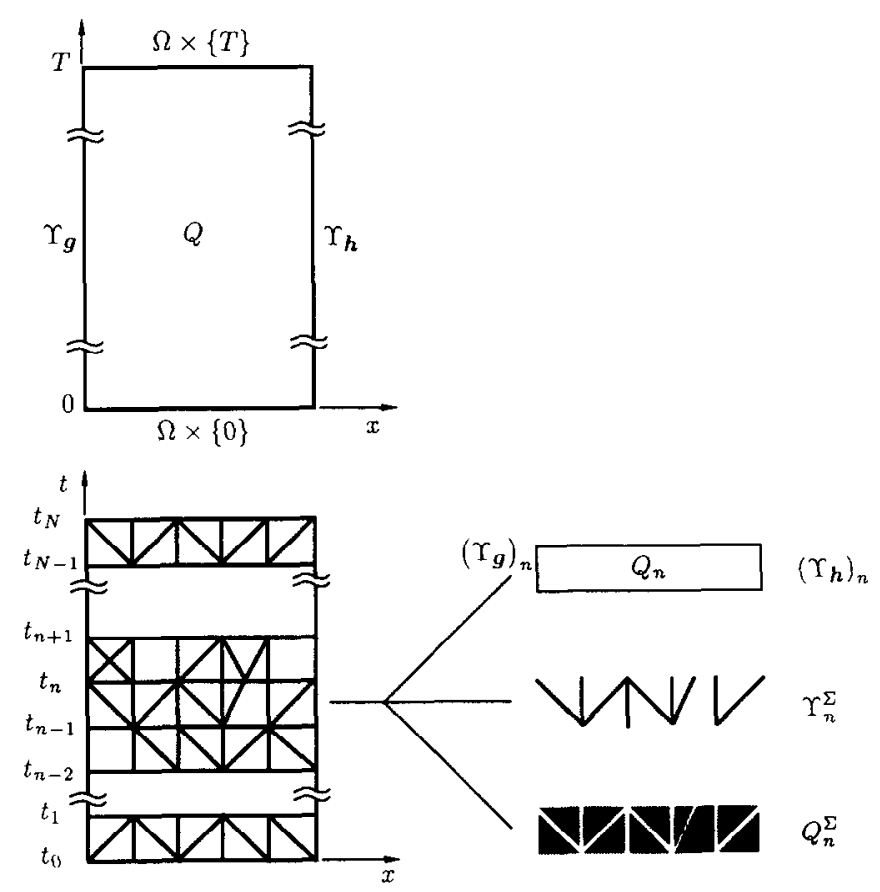

Fig. 1. Illustration of space-time finite element notation.

Assuming the function $w(t)$ to be discontinuous at time $t_{n}$, the temporal jump operator is defined by

$$
\llbracket \boldsymbol{w}\left(t_{n}\right) \rrbracket=\boldsymbol{w}\left(t_{n}^{+}\right)-\boldsymbol{w}\left(t_{n}^{-}\right)
$$

where

$$
w\left(t_{n}^{ \pm}\right)=\lim _{\epsilon \rightarrow 0 \pm} w\left(t_{n}+\epsilon\right) .
$$

The argument $\boldsymbol{x}$ has been suppressed in (18), (19) to simplify the notation.

Consider a typical space-time element with domain $Q^{e}$ and boundary $Y^{e}$. Let $n$ denote the outward unit normal vector to $Y^{e} \cap\{t\}$ in the spatial hyperplane $Q^{e} \cap\{t\}$. Consider two adjacent space-time elements. Designate one element by + and the other by $-;$ let $\boldsymbol{n}^{+}$and $\boldsymbol{n}^{-}$ denote the spatial unit outward normal vectors along the interface (see Fig. 2). To simplify the notation, the argument $t$ is suppressed. Assuming the function $\boldsymbol{w}(\boldsymbol{x})$ is discontinuous at point $\boldsymbol{x}$, the spatial jump operator is defined by

$$
\llbracket w(x) \rrbracket=w\left(x^{+}\right)-w\left(x^{-}\right),
$$

where

$$
\begin{aligned}
& w\left(x^{ \pm}\right)=\lim _{\epsilon \rightarrow 0 \pm} w(x+\epsilon n), \\
& n=n^{+}=-n^{-}
\end{aligned}
$$

The trial displacement space, $\mathscr{S}^{h}$ and the weighting function space, $\mathscr{V}^{h}$, include $k$ th-order polynomials in both $\boldsymbol{x}$ and $t$. The functions are continuous on each space-time slab, but they may be discontinuous between slabs. The collections of finite element interpolation functions are given by 


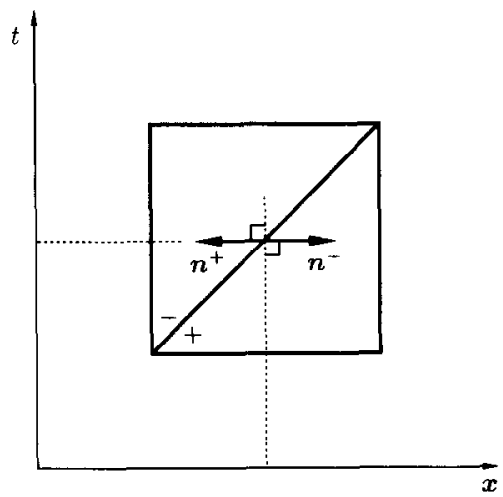

Fig. 2. Illustration of spatial outward normal vectors.

Trial displacements

$$
\mathscr{S}^{h}=\left\{\boldsymbol{u}^{h}\left|\boldsymbol{u}^{h} \in\left(\mathscr{C}^{0}\left(\bigcup_{n=1}^{N} Q_{n}\right)\right)^{d}, \boldsymbol{u}^{h}\right|_{Q_{n}^{e}} \in\left(\mathscr{P}^{k}\left(Q_{n}^{e}\right)\right)^{d}, \boldsymbol{u}^{h}=\boldsymbol{g} \text { on } Y_{\boldsymbol{g}}\right\} .
$$

Displacement weighting functions

$$
\mathscr{V}^{h}=\left\{\boldsymbol{w}^{h}\left|\boldsymbol{w}^{h} \in\left(\mathscr{C}^{0}\left(\bigcup_{n=1}^{N} Q_{n}\right)\right)^{d}, \boldsymbol{w}^{h}\right|_{Q_{n}^{e}} \in\left(\mathscr{P}^{k}\left(Q_{n}^{e}\right)\right)^{d}, \boldsymbol{w}^{h}=\mathbf{0} \text { on } Y_{g}\right\} .
$$

where $\mathscr{P}^{k}$ denotes the space of $k$ th-order polynomials and $\mathscr{C}^{0}$ denotes the space of continuous functions.

\subsection{Variational equations}

The objective is to find $u^{h} \in \mathscr{S}^{h}$ such that for all $\boldsymbol{w}^{h} \in \mathscr{V}^{h}$,

where

$$
B_{\mathrm{GLS}}\left(\boldsymbol{w}^{h}, \boldsymbol{u}^{h}\right)_{n}=L_{\mathrm{GLS}}\left(\boldsymbol{w}^{h}\right)_{n}, \quad n=1,2, \ldots, N,
$$

$$
\begin{aligned}
& B_{\mathrm{GLS}}\left(\boldsymbol{w}^{h}, \boldsymbol{u}^{h}\right)_{n}=\left(\dot{\boldsymbol{w}}^{h}, \rho \ddot{\boldsymbol{u}}^{h}\right)_{Q_{n}}+a\left(\dot{\boldsymbol{w}}^{h}, \boldsymbol{u}^{h}\right)_{Q_{n}}+\left(\mathscr{L} \boldsymbol{w}^{h}, \rho^{-1} \boldsymbol{\tau} \mathscr{L} \boldsymbol{u}^{h}\right)_{Q_{n}^{\Sigma}} \\
& +\left(\boldsymbol{n} \cdot \llbracket \boldsymbol{\sigma}\left(\nabla \boldsymbol{w}^{h}\right)(\boldsymbol{x}) \rrbracket, \rho^{-1} \boldsymbol{s} \boldsymbol{n} \cdot \llbracket \boldsymbol{\sigma}\left(\nabla \boldsymbol{u}^{h}\right)(\boldsymbol{x}) \rrbracket\right)_{Y_{n}^{\Sigma}} \\
& +\left(\boldsymbol{n} \cdot \boldsymbol{\sigma}\left(\nabla \boldsymbol{w}^{h}\right), \rho^{-1} \boldsymbol{s} \boldsymbol{n} \cdot \boldsymbol{\sigma}\left(\nabla \boldsymbol{u}^{h}\right)\right)_{\left(Y_{h}\right)_{n}} \\
& +\left(\dot{w}^{h}\left(t_{n-1}^{+}\right), \rho \dot{\boldsymbol{u}}^{h}\left(t_{n-1}^{+}\right)\right)_{\Omega}+a\left(\boldsymbol{w}^{h}\left(t_{n-1}^{+}\right), \boldsymbol{u}^{h}\left(t_{n-1}^{+}\right)\right)_{\Omega},
\end{aligned}
$$

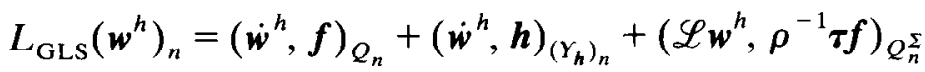

$$
\begin{aligned}
& +\left(\boldsymbol{n} \cdot \boldsymbol{\sigma}\left(\nabla \boldsymbol{w}^{h}\right), \rho^{-1} \boldsymbol{s h}\right)_{\left(Y_{h}\right)_{n}}+\left(\dot{\boldsymbol{w}}^{h}\left(t_{n-1}^{+}\right), \rho \dot{u}^{h}\left(t_{n-1}^{-}\right)\right)_{\Omega} \\
& +a\left(w^{h}\left(t_{n-1}^{+}\right), u^{h}\left(t_{n-1}^{-}\right)\right)_{\Omega},
\end{aligned}
$$

in which

$$
\mathscr{L} u=\rho \ddot{u}-\nabla \cdot \sigma(\nabla u) .
$$

With $\boldsymbol{\tau}=\boldsymbol{s}=\mathbf{0}$, the formulation is a time-discontinuous Galerkin method. The three terms evaluated over $Q_{n}$ act to weakly enforce the equation of motion, (7), over the space-time slab; terms evaluated on $\left(Y_{h}\right)_{n}$ act to weakly enforce the traction boundary condition, (9), while the terms evaluated over $\Omega$ weakly enforce continuity of displacement and velocity between slabs 
$n$ and $n-1$. The matrices $\tau$ and $s$ are called the intrinsic time scale matrix and slowness matrix, respectively (having respective dimensions of time and slowness); both are $d \times d$ matrices. The corresponding least-squares terms add stability to the underlying time-discontinuous Galerkin method without degrading its accuracy.

\section{Discontinuity-capturing operator design}

In general, least-squares operators of the form $\left(\mathscr{L} \boldsymbol{w}^{h}, \rho^{-1} \boldsymbol{\tau} \mathscr{L} \boldsymbol{u}^{h}\right)_{Q_{n}^{\Sigma}}$ provide control over a particular component of solution gradients, i.e., $\mathscr{L} \boldsymbol{u}^{h}$. The purpose of the discontinuitycapturing operator is to provide control over all components of the solution gradients. In the context of elastodynamics, first derivatives of the solution are already under control; the least-squares and discontinuity-capturing operators provide control of the second derivatives $\ddot{\boldsymbol{u}}^{h}$ and $\boldsymbol{\Delta} \boldsymbol{u}^{h}$, where $\boldsymbol{\Delta} \boldsymbol{u}^{h}=\boldsymbol{\nabla} \cdot \boldsymbol{\nabla} \boldsymbol{u}^{h}$. Defining an operator,

$$
\nabla_{x^{2}}=\left[\begin{array}{c}
\left(\partial^{2} / \partial t^{2}\right) I_{d} \\
\left(\partial^{2} / \partial x_{1}^{2}\right) I_{d} \\
\left(\partial^{2} / \partial x_{2}^{2}\right) I_{d} \\
\vdots \\
\left(\partial^{2} / \partial x_{d}^{2}\right) I_{d}
\end{array}\right],
$$

where $\boldsymbol{I}_{d}$ is the $d \times d$ identity matrix, we could attempt to control the second derivatives by including terms having the form $\nabla_{x^{2}} \boldsymbol{u}^{h} \cdot \nabla_{x^{2}} \boldsymbol{u}^{h}$. The resultant operator is dimensionally inconsistent and thus cannot be implemented in the given form. Dimensional consistency could be achieved by replacing $\nabla_{x^{2}}$ with

$$
\tilde{\boldsymbol{\nabla}}_{\boldsymbol{x}^{2}}=\left[\begin{array}{c}
\left(\partial^{2} / \partial t^{2}\right) \boldsymbol{I}_{d} \\
c^{2}\left(\partial^{2} / \partial x_{1}^{2}\right) \boldsymbol{I}_{d} \\
c^{2}\left(\partial^{2} / \partial x_{2}^{2}\right) \boldsymbol{I}_{d} \\
\vdots \\
c^{2}\left(\partial^{2} / \partial x_{d}^{2}\right) \boldsymbol{I}_{d}
\end{array}\right]
$$

where $c$ is the dilatational wave speed.

An alternative solution to the difficulty of dimensional consistency is to design the discontinuity-capturing operator to control local second derivatives of the solution. The local gradient matrix is defined by

$$
\boldsymbol{\nabla}_{\xi^{2}}=\left[\begin{array}{c}
\left(\partial^{2} / \partial \xi_{0}^{2}\right) \boldsymbol{I}_{d} \\
\left(\partial^{2} / \partial \xi_{1}^{2}\right) \boldsymbol{I}_{d} \\
\left(\partial^{2} / \partial \xi_{2}^{2}\right) \boldsymbol{I}_{d} \\
\vdots \\
\left(\partial^{2} / \partial \xi_{d}^{2}\right) \boldsymbol{I}_{d}
\end{array}\right],
$$


where $\xi_{0}, \xi_{1}, \ldots, \xi_{d}$ are the element local coordinates. The discontinuity-capturing operator is designed to control $\nabla_{\xi^{2}} \boldsymbol{u}^{h} \cdot \nabla_{\xi^{2}} \boldsymbol{u}^{h}$ and has the form

$$
\int_{Q_{n}^{e}} \boldsymbol{\vartheta} \boldsymbol{\nabla}_{\boldsymbol{\xi}^{2}} \boldsymbol{w}^{h} \cdot \boldsymbol{\nabla}_{\boldsymbol{\xi}^{2}} \boldsymbol{u}^{h} \mathrm{~d} Q
$$

where $\vartheta \geqslant 0$ and is called the viscosity coefficient. Note that the discontinuity-capturing operator is defined over each element of a space-time slab. The discontinuity-capturing formulation is given by

where

$$
B\left(\boldsymbol{w}^{h}, \boldsymbol{u}^{h}\right)_{n}=L\left(\boldsymbol{w}^{h}\right)_{n}, \quad n=1,2, \ldots, N
$$

$$
\begin{aligned}
& B\left(\boldsymbol{w}^{h}, \boldsymbol{u}^{h}\right)_{n}=B_{\mathrm{GLS}}\left(\boldsymbol{w}^{h}, \boldsymbol{u}^{h}\right)_{n}+\int_{Q_{n}^{\Sigma}} \vartheta \nabla_{\xi^{2}} \boldsymbol{w}^{h} \cdot \nabla_{\xi^{2}} \boldsymbol{u}^{h} \mathrm{~d} Q \\
& L\left(\boldsymbol{w}^{h}\right)_{n}=L_{\mathrm{GLS}}\left(\boldsymbol{w}^{h}\right)_{n},
\end{aligned}
$$

To retain the accuracy of the underlying Galerkin/least-squares formulation, the discontinuity-capturing operator must also satisfy consistency requirements; this may be accomplished by letting the viscosity coefficient be a function of the residual, i.e.,

where

$$
\vartheta=f\left(\boldsymbol{R}^{h}\right),
$$

$$
\boldsymbol{R}^{h}=\mathscr{L} \boldsymbol{u}^{h}-\boldsymbol{f}
$$

The viscosity coefficient should be proportional to some power of the residual; the rationale for this design condition is as follows. If the solution is smooth, the solution gradients and residual are small; thus, the viscosity coefficient should also be small so that the formulation is essentially equivalent to the Galerkin/least-squares method. Conversely, in regions where the solution gradients are large, the residual also is large and the viscosity coefficient should be large for the discontinuity-capturing operator to be effective. Two choices for the viscosity coefficient are presented in this paper: the 'quadratic' and 'linear' viscosity coefficients.

\subsection{Quadratic discontinuity-capturing operator}

The quadratic discontinuity-capturing operator is obtained by letting

$$
\vartheta=\frac{\left(\boldsymbol{R}^{h} \cdot \rho^{-1} \boldsymbol{\tau} \boldsymbol{R}^{h}\right)}{\left(\boldsymbol{\nabla}_{\xi^{2}} \boldsymbol{u}^{h} \cdot \nabla_{\xi^{2}} \boldsymbol{u}^{h}\right)},
$$

where $\boldsymbol{\tau}$ is the intrinsic time scale matrix of the Galerkin/least-squares formulation. Then, the quadratic discontinuity-capturing operator is given by

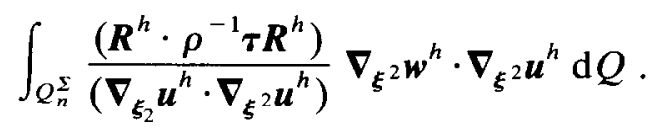

Galeão and Dutra do Carmo [9] first developed a quadratic discontinuity-capturing operator for first-order advective-diffusive problems. Shakib [11] generalized the quadratic discontinuity-capturing operator for first-order systems by using local gradients. 


\subsection{Linear discontinuity-capturing operator}

The linear discontinuity-capturing operator is defined by choosing

$$
\vartheta=\sum_{i=0}^{d} \frac{\partial \xi_{i}}{\partial t} \frac{\left(\boldsymbol{R}^{h} \cdot \rho^{-1} \boldsymbol{\tau} \boldsymbol{R}^{h}\right)^{1 / 2}}{\left(\boldsymbol{\nabla}_{\boldsymbol{\xi}^{2}} \boldsymbol{u}^{h} \cdot \rho^{-1} \boldsymbol{\tau} \boldsymbol{\nabla}_{\boldsymbol{\xi}^{2}} \boldsymbol{u}^{h}\right)^{1 / 2}} .
$$

The linear discontinuity-capturing operator is given by

$$
\int_{Q_{n}^{S}} \sum_{i=0}^{d} \frac{\partial \xi_{i}}{\partial t} \frac{\left(\boldsymbol{R}^{h} \cdot \rho^{-1} \boldsymbol{\tau} \boldsymbol{R}^{h}\right)^{1 / 2}}{\left(\boldsymbol{\nabla}_{\boldsymbol{\xi}^{2}} \boldsymbol{u}^{h} \cdot \rho^{-1} \boldsymbol{\tau} \boldsymbol{\nabla}_{\xi^{2}} \boldsymbol{u}^{h}\right)^{1 / 2}} \nabla_{\boldsymbol{\xi}^{2} \boldsymbol{w}^{h}} \cdot \boldsymbol{\nabla}_{\boldsymbol{\xi}^{2}} \boldsymbol{u}^{h} \mathrm{~d} Q .
$$

We emphasize that the discontinuity-capturing formulations are nonlinear since the viscosity coefficient depends on the residual. The labels 'linear' and 'quadratic' were chosen because of the similarity of the corresponding discontinuity-capturing operators to the linear and quadratic artificial viscosities used in finite difference methods.

In the context of fluid dynamics, the discontinuity-capturing operators of Hughes et al. [3] and Hughes and Mallet [4] are variants of linear discontinuity-capturing operators. Alternative linear discontinuity-capturing operators have been developed by Johnson and Szepessy [5-8]. Their work is particularly interesting in that it includes mathematical analyses of the resultant finite element formulations that demonstrate the need for the discontinuity-capturing operators for nonlinear problems.

\section{Stability and convergence analyses}

The discontinuity-capturing operators are designed to increase the stability of the Galerkin/ least-squares method near sharp gradients in the solution without degrading accuracy in smooth regions. Thus, one would like to prove that the discontinuity-capturing formulations are stable and have the same convergence rates as the Galerkin/least-squares formulations.

Stability and convergence of the Galerkin/least-squares method, (25), as measured in the $\||\|| \mid$ norm, were proved in [12]; the assumptions, pertinent steps and results of these proofs are repeated here as they are needed to prove stability and convergence of the discontinuitycapturing formulation.

By summing (25) over the time slabs,

where

$$
B_{\mathrm{GLS}}\left(\boldsymbol{w}^{h}, \boldsymbol{u}^{h}\right)=L_{\mathrm{GLS}}\left(\boldsymbol{w}^{h}\right),
$$

$$
\begin{aligned}
B_{\mathrm{GLS}}\left(\boldsymbol{w}^{h}, \boldsymbol{u}^{h}\right)=\sum_{n=1}^{N} & \left\{\left(\dot{\boldsymbol{w}}^{h}, \rho \ddot{u}^{h}\right)_{Q_{n}}+a\left(\dot{\boldsymbol{w}}^{h}, \boldsymbol{u}^{h}\right)_{Q_{n}}+\left(\mathscr{L} \boldsymbol{w}^{h}, \rho^{-1} \boldsymbol{\tau} \mathscr{L} \boldsymbol{u}^{h}\right)_{Q_{n}^{\Sigma}}\right. \\
& +\left(\boldsymbol{n} \cdot \llbracket \boldsymbol{\sigma}\left(\nabla \boldsymbol{w}^{h}\right)(\boldsymbol{x}) \rrbracket, \rho^{-1} \boldsymbol{s} \cdot \llbracket \boldsymbol{\sigma}\left(\nabla \boldsymbol{u}^{h}\right)(\boldsymbol{x}) \rrbracket\right)_{Y_{n}^{\Sigma}} \\
& \left.+\left(\boldsymbol{n} \cdot \boldsymbol{\sigma}\left(\nabla \boldsymbol{w}^{h}\right), \rho^{-1} \boldsymbol{s} \boldsymbol{n} \cdot \boldsymbol{\sigma}\left(\nabla \boldsymbol{u}^{h}\right)\right)_{\left(Y_{h}\right)_{n}}\right\} \\
& +\sum_{n=1}^{N-1}\left\{\left(\dot{\boldsymbol{w}}^{h}\left(t_{n}^{+}\right), \rho \llbracket \dot{\boldsymbol{u}}^{h}\left(t_{n}\right) \rrbracket\right)_{\Omega}+a\left(\boldsymbol{w}^{h}\left(t_{n}^{+}\right), \llbracket \boldsymbol{u}^{h}\left(t_{n}\right) \rrbracket\right)_{\Omega}\right\} \\
& +\left(\dot{\boldsymbol{w}}^{h}\left(0^{+}\right), \rho \dot{\boldsymbol{u}}^{h}\left(0^{+}\right)\right)_{\Omega}+a\left(\boldsymbol{w}^{h}\left(0^{+}\right), \boldsymbol{u}^{h}\left(0^{+}\right)\right)_{\Omega},
\end{aligned}
$$




$$
\begin{aligned}
L_{\mathrm{GLS}}\left(\boldsymbol{w}^{h}\right)=\sum_{n=1}^{N}\left\{\left(\dot{\boldsymbol{w}}^{h}, \boldsymbol{f}\right)_{Q_{n}}+\left(\dot{\boldsymbol{w}}^{h}, \boldsymbol{h}\right)_{\left(\Upsilon_{h}\right)_{n}}+\left(\mathscr{L} \boldsymbol{w}^{h}, \rho^{-1} \tau f\right)_{Q_{n}^{\Sigma}}\right. \\
\left.+\left(\boldsymbol{n} \cdot \boldsymbol{\sigma}\left(\nabla \boldsymbol{w}^{h}\right), \rho^{-1} \boldsymbol{s} \boldsymbol{h}\right)_{\left(Y_{h}\right)_{n}}\right\} \\
+\left(\dot{\boldsymbol{w}}^{h}\left(0^{+}\right), \boldsymbol{\rho} \boldsymbol{v}_{0}\right)_{\Omega}+a\left(\boldsymbol{w}^{h}\left(0^{+}\right), \boldsymbol{u}_{0}\right)_{\Omega} .
\end{aligned}
$$

The norm associated with (42) is defined by

$$
\begin{aligned}
&\|\| \boldsymbol{w}^{h} \|\left.\right|^{2}= \mathscr{E}\left(\boldsymbol{w}^{h}\left(T^{-}\right)\right)+\mathscr{E}\left(\boldsymbol{w}^{h}\left(0^{+}\right)\right)+\sum_{n=1}^{N-1} \mathscr{E}\left(\llbracket \boldsymbol{w}^{h}\left(t_{n}\right) \rrbracket\right) \\
&+\sum_{n=1}^{N}\left\{\left(\mathscr{L} \boldsymbol{w}^{h}, \rho^{-1} \boldsymbol{\tau} \mathscr{L} \boldsymbol{w}^{h}\right)_{Q_{n}^{\Sigma}}\right. \\
&+\left(\llbracket \boldsymbol{\sigma}\left(\nabla \boldsymbol{w}^{h}\right)(\boldsymbol{x}) \rrbracket \cdot \boldsymbol{n}, \rho^{-1} \boldsymbol{s} \llbracket \boldsymbol{\sigma}\left(\nabla \boldsymbol{w}^{h}\right)(\boldsymbol{x}) \rrbracket \cdot \boldsymbol{n}\right)_{Y_{n}^{\Sigma}} \\
&\left.+\left(\boldsymbol{\sigma}\left(\nabla \boldsymbol{w}^{h}\right) \cdot \boldsymbol{n}, \rho^{-1} \boldsymbol{s} \boldsymbol{\sigma}\left(\nabla \boldsymbol{w}^{h}\right) \cdot \boldsymbol{n}\right)_{\left(Y_{h}\right)_{n}}\right\},
\end{aligned}
$$

where, for linear elastodynamics, the total energy is defined as

$$
\mathscr{E}(\boldsymbol{w})=\frac{1}{2}(\dot{w}, \rho \dot{w})_{\Omega}+\frac{1}{2} a(w, w)_{\Omega} .
$$

The stability condition for the Galerkin/least-squares method is

$$
\left\|\boldsymbol{w}^{h}\right\|^{2}=B_{\mathrm{GLS}}\left(\boldsymbol{w}^{h}, \boldsymbol{w}^{h}\right) .
$$

A sufficiently smooth exact solution of (7)-(11) and (3) satisfies the variational equation

$$
B_{\mathrm{GLS}}\left(\boldsymbol{w}^{h}, u\right)=L_{\mathrm{GLS}}\left(w^{h}\right) \text {. }
$$

Thus, for all $w^{h} \in \mathscr{V}^{h}$,

where

$$
B_{\mathrm{GLS}}\left(\boldsymbol{w}^{h}, e\right)=0
$$

$$
\boldsymbol{e}=\boldsymbol{u}^{h}-\boldsymbol{u}
$$

Equation (49) is an appropriate consistency condition for (42).

Let $\Pi u \in \mathscr{S}^{h}$ denote an interpolant of $\boldsymbol{u}$. The error, $\boldsymbol{e}$, can be written as

where

$$
\boldsymbol{e}=\boldsymbol{e}^{h}+\boldsymbol{\eta}
$$

$$
\begin{aligned}
\boldsymbol{e}^{h} & =\left(\boldsymbol{u}^{h}-\Pi \boldsymbol{u}\right) \in \mathscr{V}^{h}, \\
\boldsymbol{\eta} & =\Pi \boldsymbol{u}-\boldsymbol{u}
\end{aligned}
$$

An appropriate space-time mesh parameter is given by

$$
h=\max \{c \Delta t, \Delta x\},
$$


where $c$ is the dilatational wave speed and $\Delta x$ and $\Delta t$ are maximum element diameters in space and time, respectively.

THEOREM 1 (Galerkin/least-squares error estimates). Assume $\tau$ and s satisfy

$$
\begin{aligned}
& c_{1} h \leqslant\|\tau\| \leqslant c_{2} h, \\
& c_{3} \leqslant\|s\| \leqslant c_{4},
\end{aligned}
$$

where $c_{1}, c_{2}, c_{3}$ and $c_{4}$ are positive constants $\left(c_{2}>c_{1}, c_{4}>c_{3}\right)$. Assuming $u \in\left(H^{k+1}(Q)\right)^{d}$, the interpolation error, $\boldsymbol{\eta}$, satisfies

$$
\begin{aligned}
& \sum_{n=1}^{N}\left(\dot{\boldsymbol{\eta}}, \rho \boldsymbol{\tau}^{-1} \dot{\boldsymbol{\eta}}\right)_{Q_{n}} \leqslant c(\boldsymbol{u}) h^{2 k-1}, \\
& \sum_{n=1}^{N}\left(\mathscr{L} \boldsymbol{\eta}, \rho^{-1} \boldsymbol{\tau} \mathscr{L} \boldsymbol{\eta}\right)_{Q_{n}^{\Sigma}} \leqslant c(\boldsymbol{u}) h^{2 k-1} \\
& \sum_{n=1}^{N}\left(\dot{\boldsymbol{\eta}}, \rho \boldsymbol{s}^{-1} \dot{\boldsymbol{\eta}}\right)_{Y_{n}^{\Sigma} \cup\left(Y_{\boldsymbol{h}}\right)_{n}} \leqslant c(\boldsymbol{u}) h^{2 k-1}, \\
& \sum_{n=1}^{N}\left\{\left(\boldsymbol{n} \cdot \llbracket \boldsymbol{\sigma}(\nabla \boldsymbol{\eta})(\boldsymbol{x}) \rrbracket, \rho^{-1} \boldsymbol{s} \boldsymbol{n} \cdot \llbracket \boldsymbol{\sigma}(\nabla \boldsymbol{\eta})(\boldsymbol{x}) \rrbracket\right)_{Y_{n}^{\Sigma}}\right. \\
& \left.\quad+\left(\boldsymbol{n} \cdot \boldsymbol{\sigma}(\nabla \boldsymbol{\eta})(\boldsymbol{x}), \rho^{-1} \boldsymbol{s} \boldsymbol{n} \cdot \boldsymbol{\sigma}(\nabla \boldsymbol{\eta})(\boldsymbol{x})\right)_{\left(Y_{h}\right)_{n}}\right\} \leqslant c(\boldsymbol{u}) h^{2 k-1} \\
& \quad \mathscr{E}\left(\boldsymbol{\eta}\left(T^{-}\right)\right)+\mathscr{E}\left(\boldsymbol{\eta}\left(0^{+}\right)\right) \\
& \quad+\sum_{n=1}^{N-1}\left\{\mathscr{E}\left(\boldsymbol{\eta}\left(t_{n}^{-}\right)\right)+\mathscr{E}\left(\boldsymbol{\eta}\left(t_{n}^{+}\right)\right)\right\} \leqslant c(\boldsymbol{u}) h^{2 k-1}
\end{aligned}
$$

where $c(u)$ is independent of $h$. Then

$$
\left.\|\boldsymbol{e}\|\right|^{2} \leqslant c(\boldsymbol{u}) h^{2 k-1}
$$

PROOF. The basic steps of the proof proceed as follows:

$$
\begin{aligned}
& \left\|e^{h}\right\|^{2}=B_{\mathrm{GLS}}\left(e^{h}, e^{h}\right) \quad \text { (by (47)) } \\
& =B_{\mathrm{GLS}}\left(e^{h}, e-\boldsymbol{\eta}\right) \quad(\text { by }(51)) \\
& =-B_{\mathrm{GLS}}\left(\boldsymbol{e}^{h}, \boldsymbol{\eta}\right) \quad(\text { by }(49)) \\
& \leqslant\left|B_{\mathrm{GLS}}\left(\boldsymbol{e}^{h}, \boldsymbol{\eta}\right)\right| \\
& \leqslant \frac{1}{2}\|\| e^{h} \|\left.\right|^{2}+c(u) h^{2 k-1},
\end{aligned}
$$

where the steps to obtain the last line of (63) have been omitted. From the interpolation estimates, (45), and the triangle inequality

$$
\left.\|\boldsymbol{e}\|\right|^{2} \leqslant\left. 2\left\|\boldsymbol{e}^{h}\right\|\right|^{2}+2\|\| \boldsymbol{\eta} \| \leqslant c(\boldsymbol{u}) h^{2 k-1},
$$

which completes the proof. 
Stability and convergence of the discontinuity-capturing formulation are proved in the \|\|$\cdot \|$ norm, (45) Summing (33) over the time slabs,

where

$$
B_{\mathrm{DC}}\left(\boldsymbol{u}^{h} ; \boldsymbol{w}^{h}, \boldsymbol{u}^{h}\right)=L_{\mathrm{DC}}\left(\boldsymbol{w}^{h}\right),
$$

$$
\begin{aligned}
& B_{\mathrm{DC}}\left(\boldsymbol{v}^{h} ; \boldsymbol{w}^{h}, \boldsymbol{u}^{h}\right)= B_{\mathrm{GLS}}\left(\boldsymbol{w}^{h}, \boldsymbol{u}^{h}\right) \\
&+\sum_{n=1}^{N} \int_{Q_{n}^{\Sigma}} \vartheta\left(\mathscr{L} \boldsymbol{v}^{h}-\boldsymbol{f}\right) \boldsymbol{\nabla}_{\boldsymbol{\xi}^{2}} \boldsymbol{w}^{h} \cdot \boldsymbol{\nabla}_{\xi^{2}} \boldsymbol{u}^{h} \mathrm{~d} Q, \\
& L_{\mathrm{DC}}\left(\boldsymbol{w}^{h}\right)=L_{\mathrm{GLS}}\left(\boldsymbol{w}^{h}\right) .
\end{aligned}
$$

Since the discontinuity-capturing terms are positive-semidefinite, the stability condition for the discontinuity-capturing formulation is easily obtained using (47) and (66); the result is

$$
\left\|\boldsymbol{w}^{h} \mid\right\|^{2} \leqslant B_{\mathrm{DC}}\left(\boldsymbol{u}^{h} ; \boldsymbol{w}^{h}, \boldsymbol{w}^{h}\right) .
$$

Error estimates are more difficult to obtain than the stability condition; clearly, the convergence proofs require an explicit form of the viscosity coefficient. The principal difficulty is showing that the discontinuity-capturing operator is sufficiently small for problems having smooth solutions. For the quadratic discontinuity-capturing formulation, we shall prove that if the solution converges at a given rate then it will converge at the optimal rate. Another interpretation of this restriction on the proof is that the residual is assumed to be bounded from above by certain powers of the mesh parameter, $h$. These results are useful in that they provide a measure of the smoothness required to prove convergence although the restriction on the residual is more stringent than desired. Numerical results presented in Section 6 demonstrate that both the linear and quadratic discontinuity-capturing formulations achieve the same rates of convergence as the Galerkin/least-squares formulation.

THEOREM 2 (Quadratic discontinuity-capturing error estimates). Assume (55)-(61) hold. Assume the following seminorm equivalences and inverse estimate hold:

$$
\begin{aligned}
& c_{1} h^{4}|\boldsymbol{w}|_{2}^{2} \leqslant\left(\nabla_{\xi^{2}} \boldsymbol{w}, \nabla_{\xi^{2}} \boldsymbol{w}\right)_{Q_{n}^{\Sigma}} \leqslant c_{2} h^{4}|\boldsymbol{w}|_{2}^{2}, \\
& c_{3}|\boldsymbol{w}|_{1}^{2} \leqslant \int_{I_{n}} \mathscr{E}(\boldsymbol{w}) \mathrm{d} t \leqslant c_{4}|\boldsymbol{w}|_{1}^{2}, \\
& |\boldsymbol{w}|_{2}^{2} \leqslant c h^{-2}|\boldsymbol{w}|_{1}^{2},
\end{aligned}
$$

where $c, c_{1}, c_{2}, c_{3}$ and $c_{4}$ are positive constants $\left(c_{2}>c_{1}, c_{4}>c_{3}\right) ;|w|_{1}$ and $|w|_{2}$ are the $H^{1}$ and $H^{2}$ seminorms, respectively. Finally, the residual is assumed to be bounded by

$$
\max _{n=1,2, \ldots, N}\left(\max _{e=1,2, \ldots,\left(n_{e 1}\right)_{n}}\left(\max _{Q_{n}^{e}}\left(\boldsymbol{R}^{h} \cdot \rho^{-1} \boldsymbol{\tau} \boldsymbol{R}^{h}\right)\right)\right) \leqslant c h^{2} .
$$

Then

$$
\|\boldsymbol{e}\| \leqslant c(\boldsymbol{u}) h^{2 k-1} .
$$

That is, the quadratic discontinuity-capturing formulation converges at the same rate as the Galerkin/least-squares method.

PROOF. Before proceeding with the proof, we note that (72) may be expressed as 


$$
\max _{n-1,2, \ldots, N}\left(\max _{c-1,2, \ldots,\left(n_{\mathrm{e} 1}\right)_{n}}\left(\max _{Q_{n}^{e}}\left(\mathscr{L} \boldsymbol{e} \cdot \rho^{-1} \tau \mathscr{L} \boldsymbol{e}\right)\right)\right) \leqslant c h^{2} .
$$

Integrating the expressions on both sides of (74) over $Q_{n}^{\Sigma}$ and summing over the time slabs yields

$$
\sum_{n=1}^{N}\left(\mathscr{L} \boldsymbol{e}, \rho^{-1} \tau \mathscr{L} \boldsymbol{e}\right)_{Q_{n}^{\Sigma}} \leqslant c T \text { Vol } h^{2},
$$

where $\mathrm{Vol}=\int_{\Omega} \mathrm{d} \Omega$. In words, (75) places a restriction on the applicability of the proof by requiring that the solution converge in the seminorm $\left(\mathscr{L} e, \rho^{-1} \tau \mathscr{L} \boldsymbol{e}\right)_{Q_{n}^{\Sigma}}$ at least at $\mathrm{O}\left(h^{2}\right)$. Note that (75) is independent of the order of the displacement interpolation, $k$.

Derivations of inverse estimates similar to (71) may be found in [14].

The following estimate is needed in the proof:

$$
\max _{n=1,2, \ldots, N}\left(\max _{e=1,2, \ldots,\left(n_{\mathrm{e}}\right)_{n}}\left(\max _{Q_{n}^{e}}\left(\frac{\boldsymbol{\nabla}_{\boldsymbol{\xi}^{2}}(\Pi \boldsymbol{u}) \cdot \boldsymbol{\nabla}_{\boldsymbol{\xi}^{2}}(\Pi \boldsymbol{u})}{\left(\boldsymbol{\nabla}_{\boldsymbol{\xi}^{2}} \boldsymbol{u}^{h} \cdot \boldsymbol{\nabla}_{\boldsymbol{\xi}^{2}} \boldsymbol{u}^{h}\right)^{2}}\right)\right)\right) \leqslant c h^{-4} .
$$

This estimate is relatively easy to prove provided that $\boldsymbol{\nabla}_{\boldsymbol{\xi}^{2}} \boldsymbol{u}^{h} \cdot \boldsymbol{\nabla}_{\boldsymbol{\xi}^{2}} \boldsymbol{u}^{h}$ is bounded away from zero. Such a check is implemented in the algorithm and so it may be assumed to be enforced. It is straightforward to show that the numerator is bounded since $\Pi u=\boldsymbol{\eta}+\boldsymbol{u}$. The $h^{4}$ factor is obtained by converting the local $(\xi)$ derivatives to global $(\boldsymbol{x})$ derivatives.

Using (48)-(53) and (65)-(67),

$$
\begin{aligned}
& B_{\mathrm{DC}}\left(\boldsymbol{u}^{h} ; \boldsymbol{e}^{h}, \boldsymbol{e}^{h}\right)
\end{aligned}
$$

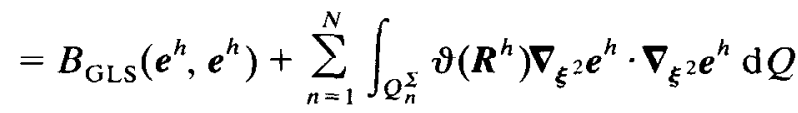

$$
\begin{aligned}
& =B_{\mathrm{GLS}}\left(\boldsymbol{e}^{h}, \boldsymbol{u}^{h}-\Pi \boldsymbol{u}\right)+\sum_{n=1}^{N} \int_{Q_{n}^{\Sigma}} \boldsymbol{\vartheta}\left(\boldsymbol{R}^{h}\right) \boldsymbol{\nabla}_{\boldsymbol{\xi}^{2}} \boldsymbol{e}^{h} \cdot \nabla_{\boldsymbol{\xi}^{2}}\left(\boldsymbol{u}^{h}-\Pi \boldsymbol{u}\right) \mathrm{d} Q \\
& =B_{\mathrm{GLS}}\left(\boldsymbol{e}^{h}, \boldsymbol{u}^{h}\right)+\sum_{n=1}^{N} \int_{Q_{n}^{\Sigma}} \vartheta\left(\boldsymbol{R}^{h}\right) \boldsymbol{\nabla}_{\xi^{2}} \boldsymbol{e}^{h} \cdot \boldsymbol{\nabla}_{\xi^{2}} \boldsymbol{u}^{h} \mathrm{~d} Q \\
& -B_{\mathrm{GLS}}\left(\boldsymbol{e}^{h}, \Pi u\right)-\sum_{n=1}^{N} \int_{Q_{n}^{\Sigma}} \vartheta\left(\boldsymbol{R}^{h}\right) \nabla_{\xi^{2}} \boldsymbol{e}^{h} \cdot \nabla_{\xi^{2}}(\Pi u) \mathrm{d} Q \\
& =L_{\mathrm{DC}}\left(\boldsymbol{e}^{h}\right)-\boldsymbol{B}_{\mathrm{GLS}}\left(\boldsymbol{e}^{h}, \Pi \boldsymbol{u}\right)-\sum_{n=1}^{N} \int_{Q_{n}^{\Sigma}} \boldsymbol{\vartheta}\left(\boldsymbol{R}^{h}\right) \boldsymbol{\nabla}_{\boldsymbol{\xi}^{2}} \boldsymbol{e}^{h} \cdot \nabla_{\boldsymbol{\xi}^{2}}(\Pi \boldsymbol{u}) \mathrm{d} Q \\
& =B_{\mathrm{GLS}}\left(\boldsymbol{e}^{h}, \boldsymbol{u}-\Pi \boldsymbol{u}\right)-\sum_{n=1}^{N} \int_{Q_{n}^{\Sigma}} \boldsymbol{\vartheta}\left(\boldsymbol{R}^{h}\right) \nabla_{\boldsymbol{\xi}^{2}} \boldsymbol{e}^{h} \cdot \nabla_{\boldsymbol{\xi}^{2}}(\Pi u) \mathrm{d} Q \\
& =-B_{\mathrm{GLS}}\left(\boldsymbol{e}^{h}, \boldsymbol{\eta}\right)-\sum_{n=1}^{N} \int_{Q_{n}^{\Sigma}} \boldsymbol{\vartheta}\left(\boldsymbol{R}^{h}\right) \boldsymbol{\nabla}_{\boldsymbol{\xi}^{2}} \boldsymbol{e}^{h} \cdot \nabla_{\boldsymbol{\xi}^{2}}(\Pi u) \mathrm{d} Q .
\end{aligned}
$$

Using (68) and (77),

$$
\begin{aligned}
\left\|\boldsymbol{e}^{h}\right\|^{2} \leqslant & B_{\mathrm{GLS}}\left(\boldsymbol{e}^{h}, \boldsymbol{e}^{h}\right) \\
& +\sum_{n=1}^{N} \int_{Q_{n}^{\Sigma}} \boldsymbol{\vartheta}\left(\boldsymbol{R}^{h}\right) \boldsymbol{\nabla}_{\xi^{2}} \boldsymbol{e}^{h} \cdot \boldsymbol{\nabla}_{\boldsymbol{\xi}^{2}} \boldsymbol{e}^{h} \mathrm{~d} Q
\end{aligned}
$$




$$
\begin{aligned}
\leqslant & \left|B_{\mathrm{GLS}}\left(\boldsymbol{e}^{h}, \boldsymbol{\eta}\right)\right| \\
& +\sum_{n=1}^{N}\left|\int_{Q_{n}^{\Sigma}} \vartheta\left(\boldsymbol{R}^{h}\right) \nabla_{\xi^{2}} \boldsymbol{e}^{h} \cdot \nabla_{\xi^{2}}(\Pi \boldsymbol{u}) \mathrm{d} Q\right|
\end{aligned}
$$

From the proof of Theorem 1,

$$
\left|B_{\mathrm{GLS}}\left(\boldsymbol{e}^{h}, \boldsymbol{\eta}\right)\right| \leqslant \frac{1}{2}\left\|\left|\boldsymbol{e}^{h} \|\right|^{2}+c(\boldsymbol{u}) h^{2 k-1} .\right.
$$

Substituting (38) into (78), the remaining term to be bounded is

$$
\sum_{n=1}^{N}\left|\int_{Q_{n}^{\Sigma}} \frac{\left(\boldsymbol{R}^{h} \cdot \rho^{-1} \boldsymbol{\tau} \boldsymbol{R}^{h}\right)}{\left(\nabla_{\boldsymbol{\xi}^{2}} \boldsymbol{u}^{h} \cdot \nabla_{\boldsymbol{\xi}^{2}} \boldsymbol{u}^{h}\right)} \nabla_{\boldsymbol{\xi}^{2}} \boldsymbol{e}^{h} \cdot \nabla_{\boldsymbol{\xi}^{2}}(\Pi \boldsymbol{u}) \mathrm{d} Q\right|
$$

Using (69)-(72) and (76),

$$
\begin{aligned}
& \left|\int_{Q_{n}^{\Sigma}} \frac{\left(\boldsymbol{R}^{h} \cdot \rho^{-1} \boldsymbol{\tau} \boldsymbol{R}^{h}\right)}{\left(\boldsymbol{\nabla}_{\boldsymbol{\xi}^{2}} \boldsymbol{u}^{h} \cdot \boldsymbol{\nabla}_{\left.\boldsymbol{\xi}^{2} \boldsymbol{u}^{h}\right)}\right.} \nabla_{\xi^{2}} \boldsymbol{e}^{h} \cdot \nabla_{\boldsymbol{\xi}^{2}}(\Pi \boldsymbol{u}) \mathrm{d} Q\right| \\
& \leqslant \frac{1}{8}\left(\boldsymbol{R}^{h}, \rho^{-1} \tau \boldsymbol{R}^{h}\right)_{Q_{n}^{\Sigma}}+2\left|\int_{Q_{n}^{\Sigma}} \frac{\left(\boldsymbol{R}^{h} \cdot \rho^{-1} \boldsymbol{\tau} \boldsymbol{R}^{h}\right)}{\left(\boldsymbol{\nabla}_{\xi^{2}} \boldsymbol{u}^{h} \cdot \nabla_{\xi^{2}} \boldsymbol{u}^{h}\right)^{2}}\left(\nabla_{\xi^{2}} \boldsymbol{e}^{h} \cdot \nabla_{\xi^{2}}(\Pi u)\right)^{2} \mathrm{~d} Q\right| \\
& \leqslant \frac{1}{8}\left(\mathscr{L} \boldsymbol{e}, \rho^{-1} \tau \mathscr{L} \boldsymbol{e}\right)_{Q_{n}^{2}} \\
& +2 \int_{Q_{n}^{\Sigma}} \frac{\left(\boldsymbol{R}^{h} \cdot \rho^{-1} \boldsymbol{\tau} \boldsymbol{R}^{h}\right)}{\left(\boldsymbol{\nabla}_{\xi^{2}} \boldsymbol{u}^{h} \cdot \nabla_{\boldsymbol{\xi}^{2}} \boldsymbol{u}^{h}\right)^{2}}\left(\nabla_{\xi^{2}} e^{h} \cdot \nabla_{\xi^{2}} e^{h}\right)\left(\nabla_{\xi^{2}}(\Pi u) \cdot \nabla_{\xi^{2}}(\Pi u)\right) \mathrm{d} Q \\
& \leqslant \frac{1}{4}\left(\mathscr{L} e^{h}, \rho^{-1} \tau \mathscr{L} e^{h}\right)_{Q_{n}^{\Sigma}}+\frac{1}{4}\left(\mathscr{L} \boldsymbol{\eta}, \rho^{-1} \tau \mathscr{L} \boldsymbol{\eta}\right)_{Q_{n}^{\Sigma}}+c h^{-2}\left(\nabla_{\xi^{2}} e^{h}, \nabla_{\xi^{2}} e^{h}\right)_{Q_{n}^{\Sigma}} \\
& \leqslant \frac{1}{4}\left(\mathscr{L} e^{h}, \rho^{-1} \boldsymbol{\tau} \mathscr{L} \boldsymbol{e}^{h}\right)_{Q_{n}^{\Sigma}}+\frac{1}{4}\left(\mathscr{L} \boldsymbol{\eta}, \rho^{-1} \boldsymbol{\tau} \mathscr{L} \boldsymbol{\eta}\right)_{Q_{n}^{\Sigma}}+c h^{2}\left|\boldsymbol{e}^{h}\right|_{2}^{2} \\
& \leqslant \frac{1}{4}\left(\mathscr{L} \boldsymbol{e}^{h}, \rho^{-1} \boldsymbol{\tau} \mathscr{L} \boldsymbol{e}^{h}\right)_{Q_{n}^{\Sigma}}+\frac{1}{4}\left(\mathscr{L} \boldsymbol{\eta}, \rho^{-1} \tau \mathscr{L} \boldsymbol{\eta}\right)_{Q_{n}^{\Sigma}}+c\left|\boldsymbol{e}^{h}\right|_{1}^{2} \\
& \leqslant \frac{1}{4}\left(\mathscr{L} \boldsymbol{e}^{h}, \rho^{-1} \tau \mathscr{L} \boldsymbol{e}^{h}\right)_{Q_{n}^{\Sigma}}+\frac{1}{4}\left(\mathscr{L} \boldsymbol{\eta}, \rho^{-1} \tau \mathscr{L} \boldsymbol{\eta}\right)_{Q_{n}^{\Sigma}}+c \int_{I_{n}} \mathscr{E}\left(\boldsymbol{e}^{h}\right) \mathrm{d} t .
\end{aligned}
$$

Summing over the time slabs,

$$
\begin{aligned}
& \sum_{n=1}^{N}\left|\int_{Q_{n}^{\Sigma}} \frac{\left(\boldsymbol{R}^{h} \cdot \rho^{-1} \boldsymbol{\tau} \boldsymbol{R}^{h}\right)}{\left(\boldsymbol{\nabla}_{\xi^{2}} \boldsymbol{u}^{h} \cdot \nabla_{\boldsymbol{\xi}^{2}} \boldsymbol{u}^{h}\right)} \nabla_{\xi^{2}} \boldsymbol{e}^{h} \cdot \nabla_{\xi^{2}}(\Pi \boldsymbol{u}) \mathrm{d} Q\right| \\
& \quad \leqslant \sum_{n=1}^{N}\left\{\frac{1}{4}\left(\mathscr{L} \boldsymbol{e}^{h}, \rho^{-1} \boldsymbol{\tau} \mathscr{L} \boldsymbol{e}^{h}\right)_{Q_{n}^{\Sigma}}+\frac{1}{4}\left(\mathscr{L} \boldsymbol{\eta}, \rho^{-1} \boldsymbol{\tau} \mathscr{L} \boldsymbol{\eta}\right)_{Q_{n}^{\Sigma}}+c \int_{I_{n}} \mathscr{E}\left(\boldsymbol{e}^{h}\right) \mathrm{d} t\right\} .
\end{aligned}
$$

The first term on the right-hand side of (82) can be subsumed by the left-hand side of (78), while the second term is bounded from above by $c(u) h^{2 k-1}$ using the interpolation error estimate (58). Thus,

$$
\left\|\boldsymbol{e}^{h}\right\| \|^{2} \leqslant c(\boldsymbol{u}) h^{2 k-1}+\sum_{n=1}^{N} c \int_{I_{n}} \mathscr{E}\left(\boldsymbol{e}^{h}\right) \mathrm{d} t .
$$


Using a Gronwall inequality [15] (also see [8]),

$$
\left.\left\|\boldsymbol{e}^{h}\right\|\right|^{2} \leqslant c(u) h^{2 k-1} .
$$

Finally, using (64) and (84),

$$
\left.\|\boldsymbol{e}\|\right|^{2} \leqslant c(\boldsymbol{u}) h^{2 k-1} .
$$

Thus, provided that (72) holds, the quadratic discontinuity-capturing formulation converges optimally; that is, at the same rate as proved for the Galerkin/least-squares formulation.

If we restrict $k=2$, then optimal convergence can be proved using a less restrictive bound than (74), i.e.,

$$
\max _{n=1,2, \ldots, N}\left(\max _{e-1,2, \ldots,\left(n_{\mathrm{e} 1}\right)_{n}}\left(\max _{Q_{n}^{e}}\left(\mathscr{L} \boldsymbol{e} \cdot \rho^{-1} \tau \mathscr{L} \boldsymbol{e}\right)\right)\right) \leqslant c .
$$

Optimal convergence is proved by obtaining bounds on the difference between solutions computed using the Galerkin/least-squares and discontinuity-capturing formulations. If (86) holds, then it can be shown that

$$
\left\|u_{\mathrm{DC}}^{h}-u_{\mathrm{GLS}}^{h}\right\| \|^{2} \leqslant c h^{3},
$$

where $\boldsymbol{u}_{\mathrm{DC}}^{h}$ and $\boldsymbol{u}_{\mathrm{GLS}}^{h}$ are the solutions computed using (33) and (25), respectively. Thus, using (62) and (87),

$$
\begin{aligned}
\left\|\boldsymbol{u}_{\mathrm{DC}}^{h}-\boldsymbol{u}\right\|^{2} & =\left\|\boldsymbol{u}_{\mathrm{DC}}^{h}-\boldsymbol{u}_{\mathrm{GLS}}^{h}+\boldsymbol{u}_{\mathrm{GLS}}^{h}-\boldsymbol{u}\right\| \|^{2} \\
& \leqslant 2\left\|\boldsymbol{u}_{\mathrm{DC}}^{h}-\boldsymbol{u}_{\mathrm{GLS}}^{h}\right\|^{2}+2\left\|\boldsymbol{u}_{\mathrm{GLS}}^{h}-\boldsymbol{u}\right\|^{2} \\
& \leqslant c h^{3} .
\end{aligned}
$$

\section{Numerical results}

To demonstrate the performance of the discontinuity-capturing formulations, we consider the impact against a rigid wall of a one-dimensional, homogeneous elastic bar (see Fig. 3). Initially, the bar is moving with a uniform speed; the left end of the bar then impacts the rigid wall. This generates a compressive stress wave in the bar that starts at the impacted end and propagates towards the free end. Attention is restricted to the time interval during which the stress wave remains compressive, that is, before the wave front reaches the free end of the bar. The bar has a length of 4; the density, area and Young's modulus have unit values; the uniform initial speed also has unit value. Each space-time slab was discretized using 200 9-noded quadrilateral space-time elements as shown in Fig. 4. The intrinsic time scale and slowness matrices used for all computations were

$$
\tau=\frac{\Delta t}{4 \sqrt{1+C^{4}}}
$$



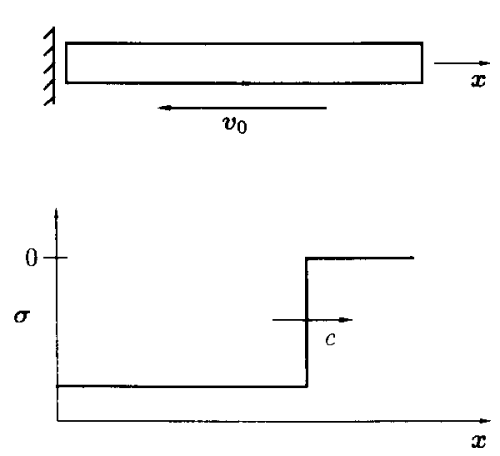

Fig. 3. One-dimensional elastic bar impact problem (top: model problem; bottom: exact solution).

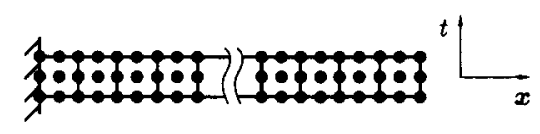

Fig. 4. Space-time finite element discretization for the bar impact problem using 2009 -noded quadrilateral elcments.

$$
s=0
$$

where $C=c \Delta t / \Delta x$ is the Courant number.

Figure 5 shows the stress distribution in the bar after the wave front has propagated through 150 elements; the solution was computed using the Galerkin/least-squares method with $C=0.5$. The discontinuity is captured quite well; the stress jump is spread over 8 elements, including the overshoot in front of and the undershoot behind the discontinuity. Because the Galerkin/least-squares algorithm is dissipative, the discontinuity will be smeared as the wave propagates through the mesh. However, the amount of smearing is small; after propagating through 50 elements, the stress jump is spread over 6 elements. That is, the stress jump has spread only one element on each side of the discontinuity after propagating through 100 additional elements.

The quadratic discontinuity-capturing formulation produces a monotone solution (see Fig. 6). Comparison of these results with the Galerkin/least-squares solution (Fig. 7) shows that the quadratic discontinuity-capturing operator maintains the accuracy of the Galerkin/leastsquares method in the smooth region as well as near the discontinuity; note that the computed slopes across the wave front are virtually identical. These results emphasize that the discontinuity-capturing formulation is a high-order accurate scheme. The solutions obtained using the quadratic and linear discontinuity-capturing operators are compared in Fig. 8

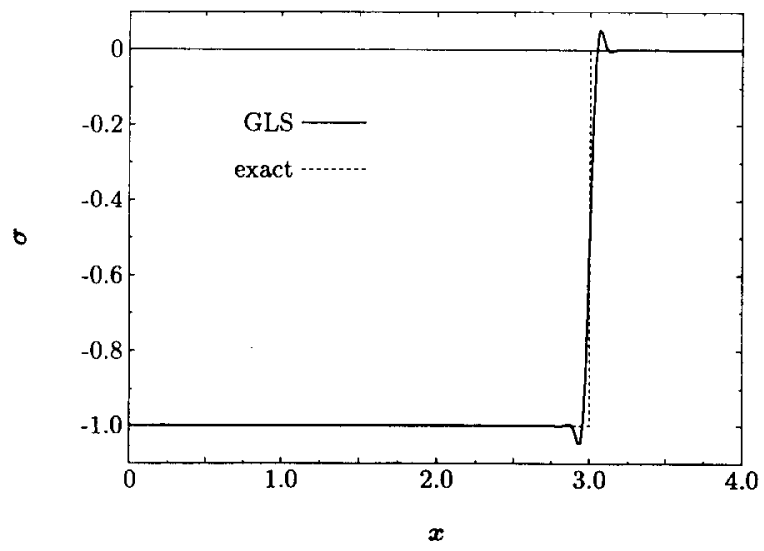

Fig. 5. Bar impact problem. Stress distribution calculated using the Galerkin/least-squares algorithm.

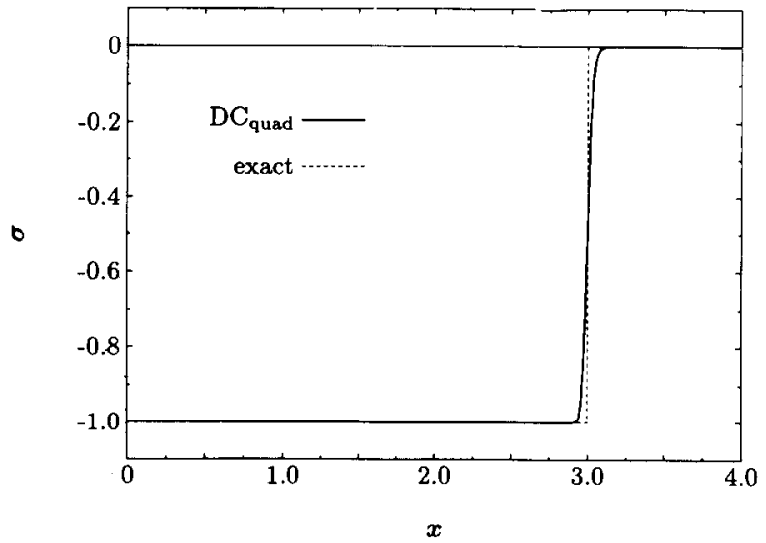

Fig. 6. Bar impact problem. Stress distribution calculated using the quadratic discontinuity-capturing algorithm. 


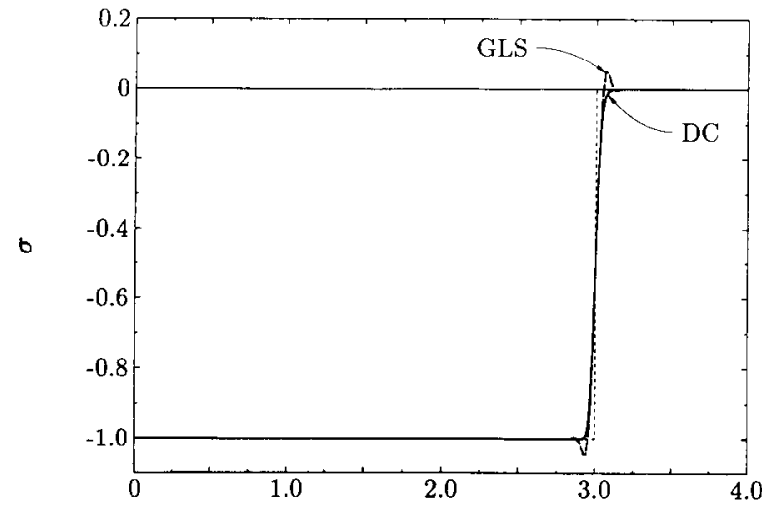

$x$

Fig. 7. Bar impact problem. Comparison of stress distribution calculated using the Galerkin/leastsquares and quadratic discontinuity-capturing algorithms.

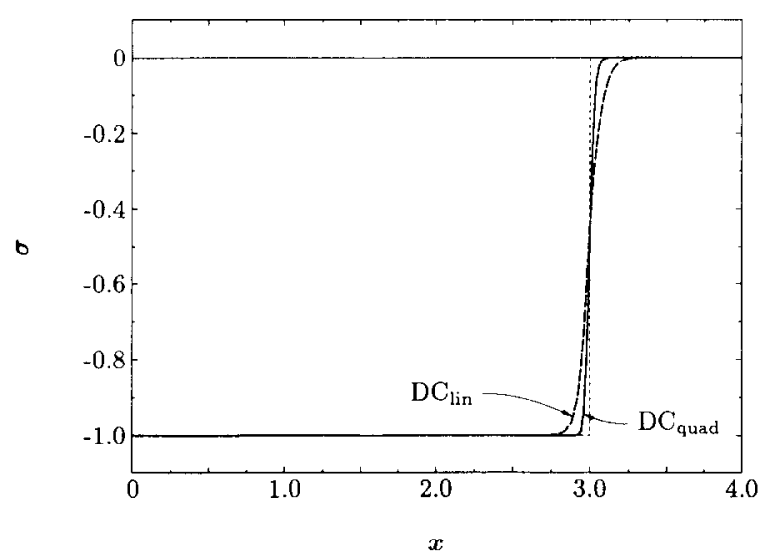

Fig. 8. Bar impact problem. Comparison of stress distribution calculated using discontinuity-capturing algorithms; $C=0.5$.

$(C=0.5)$. The linear discontinuity-capturing operator, while providing a monotone solution, is substantially more dissipative than the quadratic discontinuity-capturing operator; the stress jump is spread over 21 elements. Although the quadratic discontinuity-capturing operator performance is better than that of the linear discontinuity-capturing operator, their relative performance changes when the Courant number is increased. Figure 9 shows the stress distributions computed using $C=2.0$. The quadratic discontinuity-capturing operator response is similar to the Galerkin/least-squares response, although slightly more dissipative. The linear discontinuity-capturing operator maintains a monotone solution without increasing the spread of the stress jump, when compared to the Galerkin/least-squares results. Thus, the quadratic viscosity coefficient is too small when $C=2.0$ to control the overshoot/undershoot of the Galerkin/least-squares method. These results suggest the choice of viscosity coefficient should be based on the Courant number. Because the discontinuity-capturing operator is constructed at the element level, different discontinuity-capturing operators can be employed in different elements of the mesh. This procedure is easily implemented in a finite element code: for each element in the space-time slab, an estimate of the Courant number can be obtained; if $C \geqslant 1.0$, the linear viscosity coefficient is used, otherwise the quadratic viscosity coefficient is activated.

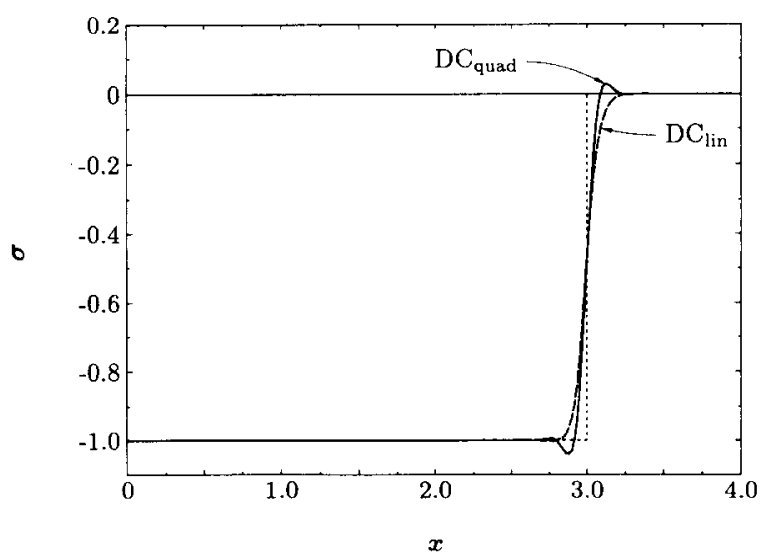

Fig. 9. Bar impact problem. Comparison of stress distribution calculated using space-time finite element methods; $C=2.0$. 


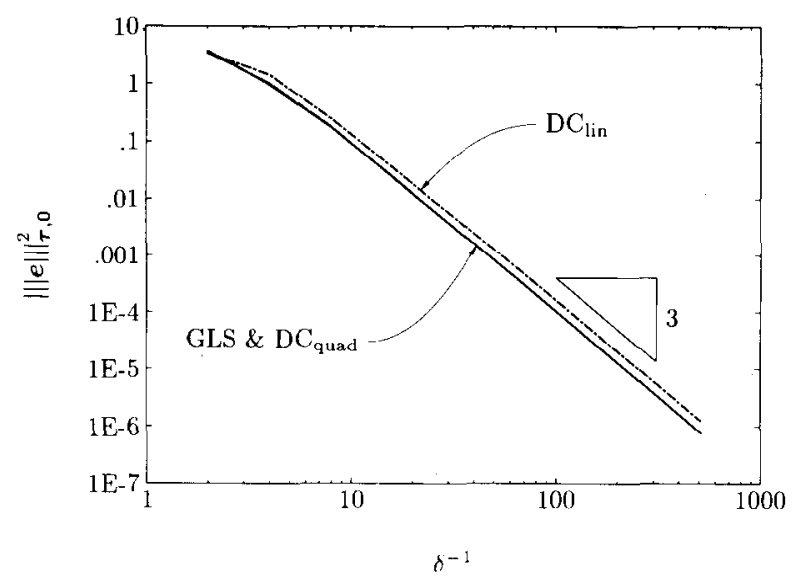

Fig. 10. Comparison of numerical error using Galerkin/least-squares (GLS), linear (DC $\mathrm{Din}_{\mathrm{in}}$ ) and quadratic $\left(\mathrm{DC}_{\mathrm{quad}}\right.$ ) discontinuity-capturing formulations; error measured in the \|\|$_{\tau, 0}$ norm; $\delta$ is the spatial distance between adjacent nodes.

To study the numerical convergence rates of the discontinuity-capturing formulations, the response of a one-dimensional, homogeneous elastic rod was calculated. Both ends of the rod were fixed, no external loads were applied, the initial velocity was zero and the initial displacement was proportional to the first harmonic. Unit values were specified for the length, area, density and elastic coefficient of the rod. The response was calculated for the time interval $0 \leqslant t \leqslant T=1.2$. The convergence rates of the Galerkin/least-squares, linear and quadratic discontinuity-capturing formulations are shown in Fig. 10. $\left(\||\cdot|\|_{\tau, 0}\right.$ denotes the norm, (45), with $s=\mathbf{0}$.) Note that the convergence rates are identical for all formulations; the linear discontinuity-capturing formulation exhibits slightly larger errors. These results suggest that a proof for convergence of the linear discontinuity-capturing formulation may be possiblc.

\section{Conclusions}

Space-time finite element methods for elastodynamics were developed that include discontinuity-capturing operators to accurately resolve sharp gradients in wave propagation problems. Control of the local second derivatives and a viscosity coefficient, defined at the element level, comprise the essential components of the proposed discontinuity-capturing operators. These operators were designed to maintain the high-order accuracy of the underlying Galerkin/least-squares finite element method; hence, they do not inherit the deficiencies of classical artificial viscosities. Stability of the new method was proved and error estimates were derived for the quadratic discontinuity-capturing formulation. Numerical results demonstrated that the quadratic discontinuity-capturing operator produces a monotone solution with excellent resolution of the discontinuity for Courant number $C=0.5$. The linear discontinuitycapturing operator was shown to be more dissipative but provided better, monotone solutions for $C=2.0$. Thus, an appropriate choice of the discontinuity-capturing operator is dependent upon the elcment Courant number; this choice may be casily implemented in a finite element program. Numerical results also demonstrated that both discontinuity-capturing formulations exhibited the same convergence rates as the Galerkin/least-squares method.

While the numerical results presented were limited to one-dimensional problems, the formulations are applicable to multidimensional problems. Of particular interest in the multidimensional problems is the possibility of waves propagating with different wave speeds. 
Generalization of the discontinuity-capturing operator may be needed to accurately resolve different wave fronts; research efforts are underway to study such problems.

\section{Acknowledgment}

Part of the research presented herein was conducted by the author at Stanford University; support from the U.S. Office of Naval Research under Contract N00014-88-K-0446 is gratefully acknowledged. The author wishes to thank T.J.R. Hughes and F. Shakib for their helpful comments and discussions.

\section{References}

[1] J. Von Neumann and R.D. Richtmyer, A method for the numerical calculation of hydrodynamic shocks, J. Appl. Phys. 21 (1950) 232-237.

[2] D. Benson, A new two-dimensional flux-limited shock viscosity for impact calculations, Comput. Methods Appl. Mech. Engrg. 93 (1991) 39-95.

[3] T.J.R. Hughes, M. Mallet and A. Mizukami, A new finite element formulation for computational fluid dynamics: II. Beyond SUPG, Comput. Methods Appl. Mech. Engrg. 54 (1986) 341-355.

[4] T.J.R. Hughes and M. Mallet, A new finite element formulation for computational fluid dynamics: IV. A discontinuity-capturing operator for multidimensional advective-diffusive systems, Comput. Methods Appl. Mech. Engrg. 58 (1986) 329-336.

[5] C. Johnson and A. Szepessy, On the convergence of streamline diffusion finite element methods for hyperbolic conservation laws, in: T.E. Tezduyar and T.J.R. Hughes, eds., Numerical Methods for Compressible Flows - Finite Difference, Element and Volume Techniques, AMD Vol. 78 (ASME, New York, 1986) $75-91$.

[6] C. Johnson and A. Szepessy, On the convergence of a finite element method for a nonlinear hyperbolic conservation law, Math. Comput. 49 (1987) 427-444.

[7] C. Johnson and A. Szepessy, Shock-capturing streamline diffusion finite element methods for nonlinear conservation laws, in: T.J.R. Hughes and T.E. Tezduyar, eds., Recent Developments in Computational Fluid Dynamics, AMD Vol. 95 (ASME, New York, 1988) 101-108.

[8] A. Szepessy, Convergence of the streamline diffusion finite element method for conservation laws, Ph.D. Thesis, Department of Mathematics, Chalmers University of Technology, Göteborg, Sweden, 1989.

[9] E.G. Dutra Do Carmo and A.C. Galeão, A consistent formulation of the finite element method to solve convective-diffusive transport problems, Rev. Brasileira Ciênc. Mec. 4 (1986) 309-340 (in Portuguese).

[10] A.C. Galeão and E.G. Dutra Do Carmo, A consistent approximate upwind Petrov-Galerkin method for convection-dominated problems, Comput. Methods Appl. Mech. Engrg. 68 (1988) 83-95.

[11] F. Shakib, Finite element analysis of the compressible Euler and Navier-Stokes equations, Ph.D. Thesis, Division of Applied Mechanics, Stanford University, Stanford, CA, 1989.

[12] T.J.R. Hughes and G.M. Hulbert, Space-time finite element methods for elastodynamics: Formulations and error estimates, Comput. Methods Appl. Mech. Engrg. 66 (1988) 339-363.

[13] G.M. Hulbert, Space-time finite element methods for second-order hyperbolic equations, Ph.D. Thesis, Division of Applied Mechanics, Stanford University, Stanford, CA, 1989.

[14] P.G. Ciarlet, The Finite Element Method for Elliptic Problems (North-Holland, Amsterdam, 1978).

[15] M.W. Hirsch and S. Smale, Differential Equations, Dynamical Systems and Linear Algebra (Academic Press, New York, 1974). 